\title{
Psychotherapie und Wissenschaft: eine Nabelschau?
}

\author{
Gerhard Burda
}

\author{
Psychotherapie-Wissenschaft 9 (2) 31-40 2019 \\ www.psychotherapie-wissenschaft.info \\ CC BY-NC-ND \\ https://doi.org/10.30820/1664-9583-2019-2-31
}

\begin{abstract}
Zusammenfassung: Der Aufsatz geht mit Bezug auf die Texte «Wissenschaftstheoretische Überlegungen zur Psychotherapiewissenschaft» (2016) und «Der Wissenschaftsbegriff der Psychotherapiewissenschaft» (2019; i.d. Heft) von Markus Erismann den beiden Fragen nach, wie Psychotherapiewissenschaft als eigenständige Wissenschaft ausgewiesen werden kann und ob der "subjektive Faktor» mit einem allgemeinen Wissenschaftsbegriff zu vereinbaren ist. Die erste Frage betreffend wird für einen externen wissenschaftstheoretischen Zugang im Vergleich zu einem aus einer Nabelschau der Psychotherapien gewonnenen Begriff von Psychotherapiewissenschaft plädiert. Die zweite Frage betreffend wird gezeigt, dass Wissenschaft prinzipiell selbst-different ist und dass auch der verpönte «subjektive Faktor» nicht aus den Wissenschaften eliminiert werden kann.
\end{abstract}

Schlüsselwörter: Psychotherapiewissenschaft, Wissenschaftstheorie, Wissenschaft, Subjekt, Selbst-Differenz

\section{Einleitung}

Ich werde mich im Folgenden nicht nur auf den neu vorliegenden Text «Der Wissenschaftsbegriff der Psychotherapiewissenschaft» von Markus Erismann (2019; i.d. Heft) beziehen, sondern zunächst auch seinen älteren Text «Wissenschaftstheoretische Überlegungen zur Psychotherapiewissenschaft» (2016) besprechen. Im Anschluss steht der aktuelle Text zur Diskussion. Gleich vorweg: Worin ich Erismann zustimme, ist erstens, dass die dringende Notwendigkeit besteht, Psychotherapiewissenschaft als eigenständige Wissenschaft auszuweisen, und dass zweitens der für die Psychotherapie zentrale «subjektive Faktor» nicht einem einseitigen, vor allem naturwissenschaftlich orientierten Wissenschaftsbegriff geopfert werden sollte. Zwei Fallstricke sind jedoch bei diesem Unterfangen zu vermeiden: Der erste ergibt sich aus einem Wissenschaftsbegriff, der aus einer Nabelschau der Psychotherapieschulen gewonnen werden soll. Statt einer Nabelschau wäre eine externe wissenschaftstheoretische bzw. -philosophische Perspektive zu überlegen. Der zweite liegt in einem unkritischen Transfer von Begriffen wie «Wissenschaft» aus der allgemeinen Wissenschaftstheorie oder «Subjekt» aus einer besonderen Philosophie. Hier wäre zu bedenken, dass Wissenschaft, die Philosophien der Wissenschaften und auch die Wissenschaftstheorie in einer Denktradition stehen, in der die conditio (in-)humana in unterschiedlichen Facetten eines Gesamtpanoramas zur Sprache kommt. Gemeint ist die Traditionslinie, die vom Mythos über die Metaphysik als erste Wissenschaft hin zu den Wissenschaften, zur Wissenschaftstheorie und letztlich auch zur Psychotherapie (und damit zur Psychotherapiewissenschaft) führt. In dieser Tradition und ihrer inhärenten Kritik haben sich im Lauf der Geschichte differenzielle Denkweisen und
Auffassungen (Selbst-Differenzen $)^{1}$ herausgebildet, die es bei Begründungs- wie Abgrenzungsfragen der Psychotherapiewissenschaft sowohl in Hinblick auf andere wissenschaftliche Disziplinen als auch in Hinblick auf das Heilverfahren Psychotherapie zu berücksichtigen gilt (s. dazu Burda, 2019). Zum Abschluss wird die interne Selbst-Differenz der Psychotherapiewissenschaft thematisiert.

\section{Wissenschaftstheoretische Überlegungen}

\section{Psychotherapie, Psychotherapiewissenschaft und Wissenschaftstheorie}

Konzentrieren wir uns zunächst auf das Verhältnis von Psychotherapie, Psychotherapiewissenschaft und Wissenschaftstheorie. Ich zitiere zunächst das Abstract von Erismanns Aufsatz "Wissenschaftstheoretische Überlegungen zur Psychotherapiewissenschaft» (2016, S. 6):

\footnotetext{
«Wissenschaftstheorie - verstanden als Reflexion auf die wissenschaftliche Tätigkeit -, Methodologie - verstanden als Reflexion auf die wissenschaftliche Verfahrensweise - und Wissenschaftsgeschichte erhöhen den Grad an Methodizität, d.h. der Selbstreflektiertheit einer bestimmten Wissenschaft, wodurch diese ein besseres Selbstverständnis gewinnt. Die vorliegenden wissenschaftstheoretischen Überlegungen sollen zeigen,
}

1 Selbst-Differenz heisst, dass jede konkrete Form von Identität immer einen gewissen Widerspruch, eine Ambivalenz, etwas Paradoxes, eine Gegenlogik oder eine Doppelung in sich enthält. In Bezug auf einen wissenschaftlichen Gegenstand zeigt sich das etwa daran, dass unterschiedliche Deutungen möglich sind, die letztlich allesamt auf einen phantasmatischen Hintergrund verweisen. 
dass die Psychotherapiewissenschaft für methodologische Selbstreflexion prädestiniert ist, welche den Grad ihrer Methodizität erhöht und zur Anerkennung als eigenständige wissenschaftliche Disziplin beiträgt. Die Geschichte der Psychotherapiewissenschaft ist gekennzeichnet durch die Bildung einer Vielzahl von Schulen und Methoden, die zum Paradigma der Psychotherapiewissenschaft gehört. Ihr fehlt aber ein schulenumfassender wissenschaftstheoretischer Rahmen, der auch ihrem interdisziplinären Charakter gerecht wird. Weil die psychotherapeutische Situation durch methodische Selbstreflexion gekennzeichnet ist, liegt es nahe, dass der Psychotherapiewissenschaftler über ein ausgeprägtes Bewusstsein der methodischen und methodologischen Selbstreflexion verfügt. Durch die Entwicklung einer eigenen Wissenschaftstheorie, Methodologie und Wissenschaftsgeschichte gelangt die Psychotherapiewissenschaft zu einem reflektierteren Selbstverständnis, das im Hinblick auf ihre Anerkennung als eigenständige Disziplin grundlegend ist.»

Die Situation, bei der es um das Verhältnis von Wissenschaftstheorie, Psychotherapieschulen und Psychotherapiewissenschaft geht, ist wie folgt:

1) Es gibt eine Vielzahl von Psychotherapieschulen.

2) Es fehlt ein umfassender wissenschaftstheoretischer Rahmen.

3) Dieser soll entwickelt werden.

4) Die Psychotherapieschulen zeichnet hohe Selbstreflexion aus.

5) Deshalb soll Psychotherapiewissenschaft für die methodische Selbstreflexivität prädestiniert sein.

6) Dies soll die Wissenschaftstheorie zeigen.

7) Eine Wissenschaftstheorie gibt es aber noch nicht.

8) Es gibt Hinweise auf die Wissenschaftstheorie von Kuhn (2017) durch das Dual paradigmatisch/präparadigmatisch.

Der Ansatz ist meines Erachtens zirkulär, er verwischt Begründungslinien sowie die Begriffe von Wissenschaftstheorie, Psychotherapiewissenschaft und Psychotherapie. Ein Satz wie: «Weil die psychotherapeutische Situation durch methodische Selbstreflexion gekennzeichnet ist, liegt es nahe, dass der Psychotherapiewissenschaftler über ein ausgeprägtes Bewusstsein der methodischen und methodologischen Selbstreflexion verfügt» (Erismann, 2016, S. 6), erklärt nicht, warum dies so sein sollte. Ist das so, weil PsychotherapeutInnen zugleich auch PsychotherapiewissenschaftlerInnen sind, wie Freuds Junktim vom Heilen und Forschen erklärt? Und wie verhält sich dies $\mathrm{zu}$ einer ebenso angesprochenen Wissenschaftstheorie? Es soll nämlich einerseits wissenschaftstheoretisch gezeigt werden, dass die Psychotherapiewissenschaft eine hohe Methodizität hat. Die entsprechende Wissenschaftstheorie soll aber erst aus der hohen Selbstreflexivität der Psychotherapieschulen gewonnen werden, für die jedoch keine gemeinsame wissenschaftstheoretische Grundlage angegeben werden kann. Was es gibt, ist ein Verweis auf Kuhn (2017): Die Psychotherapieschulen repräsentieren Kuhns prä-paradigmatisches Stadium und die Frage stellt sich, wie daraus ein psychotherapiewissenschaftlich relevantes Paradigma entstehen soll. Das Begründungsproblem verschiebt sich damit vom Verhältnis Wissenschaftstheorie zu Psychotherapiewissenschaft auf das Verhältnis von Psychotherapieschulen zu Wissenschaftstheorie. Dabei wird vieles einfach als gegeben vorausgesetzt (z. B. Begriffe wie Subjekt, Intersubjektivität, Wissenschaft).

Der Lösungsansatz ist folgender: Aus der Not wird eine Tugend gemacht, indem die Vielfalt der Psychotherapieschulen - also das Prä-Paradigmatische - einfach zum Paradigma der Psychotherapiewissenschaft erklärt wird. Dieses Paradigma - das «zentrale Verständnis des betreffenden Gegenstandsbereiches» (ebd., S. 3) - findet sich so zum Prinzip erhoben auch in der Schweizer Charta verankert. Die Frage ist nur, auf welcher Ebene das Paradigma ausgewiesen werden soll. Von der Wissenschaftstheorie kann dies nicht geleistet werden, da es sie einerseits noch nicht gibt (Punkt 7). Nimmt man andererseits an, dass auf die Kuhn'sche Wissenschaftstheorie Bezug genommen wird, dann widerspricht der Schluss von den Psychotherapieschulen gerade ob ihrer uneinheitlichen Praktiken dem behaupteten Paradigma. Folgt man Kuhn, dann müsste das Paradigma die Vielzahl der Praktiken, die für das Prä-Paradigmatische charakteristisch ist, vereinheitlichen und damit beenden. Das widerspricht aber dem Bekenntnis zur Pluralität in der Charta. Problematisch ist auch, folgt man Kuhn weiter, dass die "Annahme eines gemeinsamen Paradigmas die wissenschaftliche Gemeinschaft erst einmal von dem Zwang befreit, ihre Grundprinzipien fortgesetzt zu überprüfen» (Kuhn, 2017, S. 175). Der Grund dafür ist, dass ja genau die Selbstreflexion nicht wie bei Kuhn weniger, sondern im Gegenteil mehr werden soll. Der Gebrauch des Begriffs Paradigma ist auch insofern zweifelhaft, als ein Paradigma seinen Status deshalb erlangt, weil es «bei der Lösung einiger Probleme, welche ein Kreis von Fachleuten als brennend erkannt haben» (ebd., S. 37), erfolgreicher ist als ein mit ihm konkurrierendes. Hier drängt sich die Frage auf, ob es bei Erismann um ein Paradigma in dem Sinne gehen kann, dass ein wissenschaftliches Problem gelöst werden soll. Der Bezug scheint nicht einer wissenschaftlichen Problemlösung geschuldet zu sein, sondern eher einer gewissen Notlage, die mehr mit brennenden berufspolitischen Fragen denn mit wissenschaftlichen Problemen zu tun hat. Kuhn ist also nicht unbedingt die beste Referenz für das Unterfangen.

Kehren wir noch einmal zum Lösungsansatz zurück, bei dem einerseits das Besondere bzw. der gemeinsame Nenner des Besonderen zum Prinzip erhoben (Punkt 4) und andererseits das Fehlen eines Prinzips beklagt wird (Punkt 2 bzw. 7). Alles hängt an der Frage, auf welcher hierarchischen Ebene die wesentliche Entscheidung getroffen wird, dass die Vielzahl der Psychotherapieschulen mit ihren impliziten Welt- und Menschenbildern bzw. ihren aus Natur- und Geisteswissenschaften stammenden Auffassungen von Wissenschaft das Paradigma der Psychotherapiewissenschaft darstellen sollen. Eigentlich müsste dies doch mit Referenz auf die Wissenschaftsthe- 
orie geklärt werden: Es soll schliesslich wissenschaftstheoretisch «gezeigt werden, dass die Psychotherapiewissenschaft für methodologische Selbstreflexion prädestiniert ist» (Erismann, 2016, S. 6; Punkt 5). Nur: Es gibt weder die Psychotherapiewissenschaft als solche (Punkte 2; 6) noch - sieht man von dem bereits abgehandelten Hinweis auf Kuhn ab - eine Wissenschaftstheorie (Punkt 7). Beide werden eigentlich erst aus der Vielfalt bzw. aus der hohen methodischen Selbstreflexivität der Psychotherapieschulen rekrutiert (Punkte 4; 5).

Doch worauf reflektieren die Psychotherapieschulen tatsächlich? Reflektieren sie tatsächlich die eigenen Voraussetzungen ihrer Praxis und Theorie? Geht ihre Selbstreflexivität tatsächlich auf sich selbst im Sinne eines Verhältnisses von einer (Meta-)Theorie 2. Ordnung auf eine Theorie 1. Ordnung? Die Selbstreflexivität bezieht sich doch in erster Linie auf den therapeutischen Prozess und die Applikation einer Methode. Das dahinterstehende wissenschaftliche Verständnis stammt jedoch wieder aus jenen Wissenschaften, von denen man sich eigentlich abgrenzen möchte - also aus der Naturwissenschaft bzw. Geisteswissenschaft. (Wenn man hier in Betracht zieht, dass in manchen Staaten ohnehin nur ÄrztInnen und PsychologInnen Psychotherapie anbieten dürfen, dann entfällt auch der Bezug auf die Geisteswissenschaften.) Es gibt somit keine neutrale metatheoretische Basis, die die Selbstreflexivität der Psychotherapieschulen anleiten könnte. Diese wird erst nachträglich auf die Psychotherapiewissenshaft projiziert, das heisst, erst nachdem diese zum Träger des versammelnden Paradigmas hochstilisiert wurde. Worin gründet sich nun die Selbstreflexivität der so hochstilisierten Psychotherapiewissenschaft? Aus der angegebenen wissenschaftstheoretischen Reflexion, für die die Psychotherapieschulen via Vermittlung durch die Psychotherapiewissenschaft selbst die Basis geliefert haben. Die Psychotherapiewissenschaft soll daraus eine eigene Wissenschaftstheorie generieren, die ihr wiederum dabei helfen könnte, als wissenschaftliche Disziplin anerkannt zu werden.

Kurzum: Wenn wissenschaftstheoretisch gezeigt werden soll, dass Psychotherapiewissenschaft eine hohe Methodizität hat, dann stammt diese Zuschreibung aus der gesammelten Selbstbeschreibung der Arbeitsweise der Psychotherapieschulen. So wird eine eigene Wissenschaftstheorie gebildet, die zugleich zeigen können soll, dass die allgemeine, also nicht-schulenspezifische Psychotherapiewissenschaft für methodische Selbstreflexivität prädestiniert sein soll - ein klassischer GegenstandMethode-Zirkel. Selbstreflexivität heisst hier buchstäblich, dass man sich in einer Art wissenschaftlicher Nabelschau in sich selbst reflektiert.

Dazu eine kleine Ergänzung: Als Leitdisziplin für die hohe Selbstreflexivität wird von Erismann die Psychoanalyse genannt. Gewährsmänner sind Habermas (1975, S. 262), der schreibt, dass die Psychoanalyse als das «einzige greifbare Beispiel einer methodische Selbstreflexion in Anspruch nehmende[n] Wissenschaft relevant» ist, und Wagner (1996, S. 235), für den die Psychoanalyse die Schule «mit der längsten Tradition der Auseinander- setzung mit wissenschaftstheoretischen Fragestellungen ist». Sehen wir hier einmal von kritischeren Stimmen wie Popper (1979) ab: Bei der Psychoanalyse wiederholt sich die externe Vielfalt der Psychotherapieschulen insofern schulenintern, als es eine Vielzahl miteinander in Konflikt stehender psychoanalytischer Richtungen gibt, die einander gegenseitig den Vorwurf der Unreflektiertheit machen. Als Beispiele erwähne ich hier etwa Lacans Auseinandersetzung mit Klein oder die Debatte zwischen Freud und Jung. Folgt man Kuhn, so müsste die Erwähnung der Psychoanalyse als Leitdisziplin dieser ausserdem eine besondere «Reife» attestieren. Das hiesse dann aber, dass die Psychoanalyse nicht mehr dem prä-paradigmatischen Stadium der anderen Psychotherapie-Schulen angehören dürfte, da Reife bereits die paradigmatische Phase charakterisiert.

\section{Der fehlende Blick von aussen}

Was also fehlt, ist das differenzierende Aussen, das triangulierende Dritte, das die Selbstidentität von Psychotherapieschulen-Wissen, Psychotherapiewissenschaft und besonderer Wissenschaftstheorie zugleich aufbrechen und auf einem gemeinsamen Boden miteinander in Verbindung halten könnte. Der Verweis auf den Unterschied zwischen einer allgemeinen Wissenschaftstheorie, die auf die «Natur der Wissenschaft» gerichtet ist, und einer speziellen Wissenschaftstheorie (der Psychotherapiewissenschaft), die sich selbst zugleich mit den sich aus der Praxis «ergebenden methodologischen Entscheidungen» hinsichtlich der «Konstitutionsbedingungen» ihres Gegenstandes reflektieren können soll, ist hier zu wenig. Diese trennend-verbindende Position fehlt im Verhältnis von Psychotherapieschulen, Psychotherapiewissenschaft und Wissenschaftstheorie. Diese Funktion müsste eigentlich von aussen, das heisst von einem eigenen wissenschaftstheoretischen bzw. -philosophischen Ansatz, kommen.

Kurt Greiner von der Sigmund Freud PrivatUniversität Wien ist im Vergleich dazu wesentlich klarer, was das Verhältnis von Psychotherapie, Psychotherapiewissenschaft und Wissenschaftstheorie betrifft: Auch für ihn ist die Psychotherapie bereits eine Wissenschaft, die von der jeweiligen psychotherapeutischen Community akzeptiert wird. Dadurch wird sie erst für die Wissenschaftstheorie interessant. Sein Hintergrund ist der Konstruktive Realismus (CR) Friedrich Wallners, der das philosophische Fundament der Psychotherapiewissenschaft und die methodische Grundidee (Strangification) für den von ihm und Martin Jandl entwickelten Therapieschulendialog (TSD) liefert. Im Unterschied zu Erismann kommt dabei das wissenschaftstheoretische Moment nicht von innen - also aus den Psychotherapieschulen -, sondern von aussen, nämlich aus dem Konstruktiven Realismus. Von einer Wissenschaft - in unserem Fall einer bestimmten Psychotherapie-Richtung - ist also zunächst im Vorfeld zu verlangen, dass sie adäquat funktioniert (Funktionsaspekt, FA), um dann ihr Funktionieren kritisch reflektieren zu können (Reflexionsaspekt, RA). Erst dann, 
"wenn FA und RA gegeben sind, schafft sie wissenschaftliches Wissen. Ob die Wissenschaft gemäß ihren Zielvorgaben funktioniert, kann nur ihre Scientific Community feststellen, nicht aber die WST [Wissenschaftstheorie]: Die Frage nach dem FA ist irrelevant für WST. Wenn Wissenschaft adäquat funktioniert, schafft sie Wissen darüber, mit welchen Methoden Probleme und Fragen gelöst werden können - und nur dann wird sie interessant für WST. Ihre Aufgabe ist, der Wissenschaft methodische Hilfestellung für den RA zu liefern. Wenn die kritische Reflexion, also der interpretatorische, sinn-verstehende, hermeneutische Blick auf Bedingungen und Voraussetzungen funktioniert, dann schafft Wissenschaft auch reflexives Wissen (RA)» (Greiner, 2012, S. 50).

Bei Greiner, der selbst nicht Psychotherapeut ist, ist das Verhältnis von Psychotherapieschulen, Psychotherapiewissenschaft und Wissenschaftstheorie viel klarer differenziert als bei Erismann: Der Konstruktive Realismus liefert die wissenschaftstheoretische Begründung der Psychotherapiewissenschaft sowie die allgemeine Methodik, die in der Psychotherapiewissenschaft - etwa in Form des Therapieschulendialogs - dazu genutzt werden kann, die Psychotherapieschulen in ihrem Bestreben nach Reflexion (RA) zu unterstützen. Die Psychotherapiewissenschaft ist hier eine Art Mittler zwischen Wissenschaftstheorie (CR) und den Psychotherapieschulen, die jener bei der Herstellung reflexiven/selbstbezüglichen Therapiewissens unterstützen kann. Begründet wird dies mit Wittgensteins Sprachspieltheorie: Wer immer ein wissenschaftliches, künstlerisches, alltagssprachliches Sprachspiel spielt, kann sich «innerhalb dieser speziellen Form des Sprachgebrauchs nicht in einem hinreichenden Ausmaß klar machen, was er tatsächlich tut, weil er hierbei bestimmte Regelvorgaben befolgt und insofern auf diesen Regelgebrauch auch nicht zu blicken vermag» (Greiner, 2013, S. 5). Für ein wissenschaftliches Sprachspiel bedeutet dies, dass es einen Forschungsgegenstand in einem bestimmten Sinn strukturiert, obne den Argumentationszusammenhang von sich aus verstehen und kritisch reflektieren zu können. Der Konstruktive Realismus beharrt hier auf einer dazu nötigen Distanz, also auf einer externen Perspektive. Genau diese vermisst man bei Erismann, wodurch die Psychotherapie, Psychotherapiewissenschaft und Wissenschaftstheorie in einer diffusen Verbindung miteinander verschmolzen bleiben.

\section{Zur Selbst-Differenz von Wissenschaft und Subjektivität}

Damit komme ich zum zweiten Text Erismanns (2019), in dem es um den Aufweis einer Konkordanz zwischen dem Wissenschaftsbegriff der Psychotherapiewissenschaft und einem "allgemein anerkannten Wissenschaftsbegriff» geht. Erismann unterscheidet dabei das «Verständnis einer Wissenschaft von Wissenschaft» und das "Selbstverständnis dieser Wissenschaft als Wissenschaft». Also: Was versteht eine Wissenschaft unter Wissenschaft? bzw.
Wie versteht sie sich selbst als Wissenschaft? Diese Unterscheidung erinnert an Fragestellungen aus dem ersten Text: Ist gemeint, was Psychotherapie unter Wissenschaft versteht bzw. wie sie sich selbst als Wissenschaft erfasst? Oder geht es um Psychotherapiewissenschaft als eigenständige wissenschaftliche Disziplin? Der Autor meint wohl Ersteres: Es geht um Psychotherapie nicht nur als spezifisches Heilverfahren, sondern um Psychotherapie als Wissenschaft (was ich im Folgenden als PsychotherapieWissenschaft im weiteren Sinne bezeichnen möchte, um den Unterschied zur theoretischen Disziplin Psychotherapiewissenschaft im engeren Sinne zu unterstreichen). Diese Vermutung stützt sich auf die Fragestellung, wie die für das Selbstverständnis von Psychotherapie als Wissenschaft wichtigen Faktoren des Subjektiven, Individuellen und Widersprüchlichen im Vergleich mit den allgemeinen wissenschaftlichen Kriterien wie Objektivität, Überprüfbarkeit und Intersubjektivität einzuschätzen sind. Nun, diese Problematik stellt sich eigentlich nur für eine um Konkordanz bemühte Psychotherapie-Wissenschaft, die diese Frage von der Psychotherapie ausgehend aufwirft. Für die Psychotherapiewissenschaft im engeren Sinne stellt sich diese Problematik nur indirekt, da die Frage nach ihrer Wissenschaftlichkeit eine andere ist. Selbst wenn man bedenkt, dass zwischen Psychotherapie und Psychotherapiewissenschaft ein asymmetrisches Abhängigkeitsverhältnis besteht - ohne Psychotherapie gäbe es den Diskurs über Psychotherapie nicht -, könnte Psychotherapiewissenschaft es beim blossen Aufweis belassen, dass eine Diskrepanz dieser Art besteht. Sie könnte natürlich auch die Frage nach der Konkordanz stellen und damit die Reflexionsleistung der Psychotherapie-Schulen unterstützen, müsste dafür allerdings einen anderen (metatheoretischen) Rahmen zur Verfügung haben, der etwa imstande wäre, das Verhältnis des Physischen der Psyche und des Psychischen des Physischen zu reflektieren. ${ }^{2}$ Mit dieser Reflexion ginge es dann nicht bloss um eine Annäherung eines an der Psyche orientierten Heilverfahrens an einen naturwissenschaftlich formatierten Wissenschaftsbegriff, sondern legitimer Weise auch um die Frage, welche der Psyche zuzurechnenden Faktoren einen Wissenschaftsbegriff dieser Art eigentlich erst generieren und unterstützen (freilich ohne dabei in einen Psychologismus abzugleiten).

Damit ginge es auch um die Schaffung eines Bewusstseins für das epistemisch Unbewusste im Sinne der unreflektierten (phantasmatischen) Vorannahmen nicht nur des eigenen, sondern auch des naturwissenschaftlich orientierten Wissenschaftsbegriffes. Auf Fragen dieser Art komme ich nachfolgend in Zusammenhang mit dem Leib-SeeleProblem noch zurück. Wenden wir uns nun wieder dem Ausgangstext zu. Um das wissenschaftliche Selbstverständnis der Psychotherapie-Wissenschaft darzustellen, verweist Erismann (2019, S. 14) mit Buchmann und Kollegen (1996, S. 104) auf die «besondere Forschungsmethode,

2 In diesem Zusammenhang möchte ich auf die persönliche Erfahrung mit Studierenden der Psychotherapiewissenschaft verweisen, die völlig ratlos auf die Frage reagieren, was eigentlich mit Psyche gemeint sein könnte. 
die der Psychotherapiewissenschaft ihre Eigenständigkeit verleiht». Diese liegt in der interaktiven Datenerhebung und Untersuchung während der Therapie, die von einer systematischen und kritischen Reflexion flankiert wird. Psychotherapie wird als intersubjektives Geschehen ausgewiesen, wobei das willkürlich anmutende Moment des Subjektiven durch empathische Perspektivenübernahme und permanente Selbstreflexion minimiert werden soll. Subjektives Erleben und Wahrnehmen der objektiven Realität werden damit auf einer «höheren Ebene» integriert (Barwinski, 2017, S. 23). In der Subjekt-Subjekt-Beziehung konstituiert sich im Dialog eine intersubjektive Bedeutungsstruktur (Wagner, 1996, S. 230).

Wie im ersten findet sich also auch hier im zweiten Text der Bezug auf das «selbstreflexive Selbstbewusstsein» als entscheidende, die Wissenschaftlichkeit garantierende "qualitative Forschungsmethode» (Erismann, 2019, S. 14f). Die kritische Reflexion des Erlebens bezieht sich auf die Theorie, auf das «hermeneutische Erfassen der Individualität des Seelischen» (Wagner, 1996, S. 244; zit. n. Erismann, 2019, S. 16), das nach Regelhaftigkeiten untersucht und qualitativ interpretiert wird; die Selbstreflexion wird auch bei PatientInnen angeregt, die Ergebnisse können validiert werden. Objektivität, Überprüfbarkeit und Intersubjektivität sind damit für Erismann gegeben und stellen keinen Widerspruch zum Subjektiven und Individuellen dar. Was noch offen bleibt, ist die Widersprüchlichkeit bzw. Ambivalenz, die Erismann (2019, S. 15) mit Bezug auf die «Dialektik des Selbstbewusstseins» von Fischer (2008) als «produktive Entwicklungsfaktoren» für den Erkenntnisprozess ausweist. Trotz der Abweichungen des Wissenschaftsbegriffs der Psychotherapiewissenschaft ist damit eine Übereinstimmung mit der «allgemein anerkannten Auffassung von Wissenschaft möglich» (ebd., S. 14). Nun, ich möchte meine Bedenken aus dem ersten Teil hier nicht mehr wiederholen. Statt zu zeigen, dass der Wissenschaftsbegriff der Psychotherapiewissenschaft trotz Abweichungen von anerkannten Auffassungen von Wissenschaft mit diesen zu vereinbaren ist, möchte ich den alternativen Gedankengang verfolgen, inwiefern jeder Wissenschaftsform erstens eine inhärente Selbst-Differenz zu eigen ist und inwiefern zweitens Wissenschaft prinzipiell nicht ohne einen «subjektiven Faktor» auskommen kann. Diese Argumentation liesse sich sowohl in der Begründung von Psychotherapiewissenschaft als auch in der Auseinandersetzung mit anderen Wissenschaften geltend machen.

Am Beispiel der Wissenschaftstheorie kann beides gezeigt werden: die inhärente Selbst-Differenz und das nicht auszublendende subjektive Moment. Wissenschaftstheorie etablierte sich erst in der ersten Hälfte des 20. Jahrhunderts als eigene philosophische Disziplin und hatte ihre Blütezeit in den 70er und 80er Jahren. Angeregt durch die politischen Umbrüche zu Beginn des 20. Jahrhunderts und die bahnbrechenden Erkenntnisse in der Physik wurden in Wien und Berlin wissenschaftstheoretische Ansätze erarbeitet, deren Ziel eine Einheitswissenschaft war. Vorbild war dabei die Physik, die als beispielhaft galt. Zu nennen sind hier der Wiener Kreis mit Schlick, Carnap,
Neurath und anderen. Man strebte an, den ontologisch (metaphysisch) verstandenen Physikalismus, Naturalismus oder Materialismus als nicht empirisch einholbar und damit als sinnlos auszuweisen.

Diesen ersten Wissenschaftstheoretikern ging es um Geltungsansprüche von Aussagen, die nur so weit anerkannt werden sollten, als sie dem rekonstruierten Argumentationsmuster der Physik folgen. Die Mittel der Wahl waren logische Analysen, formale Logik und die neu entstehende Sprachphilosophie. Philosophie sollte derart zur Wissenschaftslogik (Carnap) werden. Es zeigte sich jedoch bald, dass wissenschaftstheoretische Programme selbst auf etwas beruhen, dem sie entsagen wollten - nämlich auf Fantasien über das Verbältnis von Wirklichkeit und Wissenschaft -, also auf etwas, das der Metaphysik als haltloses Spekulieren vorgeworfen wurde. So lassen sich etwa theoretische Begriffe nicht einfach formal-logisch begründen und haben selbst keine empirische Bedeutung. Sie sollen diese nur in Verbindung mit anderen theoretischen Begriffen auf dem Weg der «prognostischen Relevanz» bekommen. Die prognostische Relevanz wiederum lässt sich ausschliesslich mit syntaktischen bzw. semantischen Mitteln nicht ermitteln und muss auf pragmatische Mittel zurückgreifen (Kötter, 2017). Oder, wie Neurath es selbst ausdrückte: Sätze können wiederum nur mit Sätzen verglichen werden, nicht aber mit der Realität. Mit der Abwendung des Logischen Empirismus von der Metaphysik wurde - und dies ist eine folgenschwere Entscheidung, die für unser Thema relevant ist - das Verhältnis von Innen- und Aussenwelt zu Fall gebracht. Obwohl etwas später mitunter doch versöhnliche Töne angeschlagen wurden (etwa Popper), hat das moderne wissenschaftstheoretische Denken bis heute die Tendenz beibehalten, metaphysischen Fragen tunlichst aus dem Weg zu gehen, um die Innenwelt physikalistisch auffassen und den Schwerpunkt eher auf die Methodologie legen zu können. Dass gerade diese anti-metaphysische, gegen jeden Dogmatismus in Begründungsfragen gerichtete Denkweise genau jenen dogmatischen Anspruch selbst inszeniert, hat die Geschichte der Wissenschaftstheorie längst erwiesen. ${ }^{3}$ So wurden vor allem die beiden Grundthesen des Empirismus - «I) Die Sprache der Naturwissenschaften lässt sich so aufbauen, dass sie nur deskriptive Terme enthält, die durch Bezugnahme auf Erfahrungen interpretiert sind. II) Alle wahren naturwissenschaftlichen Behauptungen lassen sich durch Beobachtungen und nur durch Beobachtungen begründen» (v. Kutschera, 1972, S. 475) - in der auf den Logischen Empirismus folgenden wissenschaftstheoretischen Diskussion an ihre Grenze geführt. Ich erwähne hier besonders Wolfgang Stegmüller, der Ansätze von Kuhn, Lakatos und Sneed kombiniert. Um

3 Titulierte etwa Carnap Metaphysik als «inadäquates Ausdrucksmittel für das Lebensgefühl» und bezeichnete den Metaphysiker als «Musiker ohne musikalische Fähigkeiten», der im Gegensatz zum Musiker sein Lebensgefühl nicht ausdrücken kann, so erwies sich gerade sein bevorzugtes Ausdrucksmittel, die Logik, als ebenso aporetisch und totalitär in ihrem Anspruch, die Sprache der Wissenschaft zu repräsentieren. 
es kurz zu machen: Es sind besonders zwei Momente, die zeigen, dass es mit reiner Empirie nicht getan ist.

Das erste betrifft die Trennung von Theorie und empirischem Gehalt, das zweite die Trennung von Genese und Geltung. Sneed zeigte in The Logical Structure of Mathematical Physics (1971), dass eine Theorie nicht mehr als bruchloser Zusammenhang von Sätzen mit hypothetisch-empirischem Charakter aufgefasst werden kann. Er unterschied die physikalische, aus einer axiomatischen Struktur bestehende Theorie und die Menge von Modellen, die diese Struktur belegen; aus dieser sollen solche ausgewählt werden, die empirisch interpretiert werden können. Sneeds Modell erlaubte damit erstmalig eine Unterscheidung von (physikalischer) Theorie und dem empirischen Gehalt dieser Theorie.

Das zweite Moment ergab sich durch den Wechsel vom methodologischen Induktivismus des 17., 18. zum hypothetisch-deduktiven Rationalismus des 19. Jahrhunderts. Strebte Wissenschaft im 17., 18. Jahrhundert an, Phänomene sachangemessen, das heisst obne subjektive Einflüsse oder Vorlieben wiederzugeben, so brachte der Wechsel mit sich, dass nun methodologische Bedingungen erst bei der fertigen Hypothese zum Tragen kommen sollten und nicht bereits bei der Hypothesenbildung. Grund für diese methodische Umwälzung war, dass man zunehmend gewahr wurde, dass sich Wissenschaft keinesfalls auf Hypothesen beschränken liess, für die Beobachtungsbezüge feststellbar waren (siehe Elektrodynamik, Lichtwellen etc.). Dadurch ergibt sich, dass die Geltungsansprüche von $\mathrm{Hy}-$ pothesen tiefer angesetzt werden können als bisher, da sie nicht länger mit Daten gestützt sein müssen. Dieser Ansatz erweiterte somit das Spektrum zulässiger Hypothesen. Es entstanden aber auch neue Probleme, die in Hinblick auf unsere Thematik nicht uninteressant sind. Das erste Problem ist die sogenannte Duhem-Quine-Unterbestimmtheit, das zweite die Kuhn-Unterbestimmtheit (s. dazu Carrier, 2009, auf den ich mich im Folgenden beziehe).

Die Duhem-Quine-Unterbestimmtheit besagt, dass jede gegebene Menge von Erfahrungsbefunden durch unterschiedliche, begrifflich unvereinbare Erklärungssätze wiedergegeben werden kann. Die Betrachtung solcher Alternativen zeigt, dass Logik und Erfahrung allein nicht einen einzigen Denkansatz als gerechtfertigt oder wahr auszeichnen können, sondern dass zusätzliche Kriterien für die Auswahl eines Ansatzes aus der Klasse empirisch äquivalenter Alternativen erforderlich sind. Die Möglichkeit einer alternativen Theoriewahl sprengt das einfach hypothetisch-deduktive Schema. Die hypothetisch-deduktive Bestätigung kann nämlich nicht sicherstellen, dass Hypothesen mit zutreffenden Erfahrungsfolgen gültig sind. Sie beinhaltet den logischen Fehlschluss von der Bestätigung der Konsequenz auf die Richtigkeit der Prämisse, die sich dadurch zeigt, dass alternative Annahmen zu gleichen Ergebnissen bzw. Folgen führen können. Die Auswahl aus der Klasse empirisch äquivalenter Alternativen muss deshalb nicht-empirische Ansprüche heranziehen.

Nach Kuhn (2017) wird eine Hypothese anhand von nicht-empirischen Ansprüchen aus einer Liste epistemischer Ansprüche wie Genanigkeit, Widerspruchsfreiheit,
Breite des Anwendungsgebietes und Fruchtbarkeit beurteilt. Doch selbst dann, wenn nicht-empirische Kriterien zusätzlich zur empirischen Übereinstimmung in Betracht gezogen werden, ist die Theoriewahl immer noch nicht eindeutig, da wiederum eine Mehrzahl von Beurteilungskriterien möglich ist.

Beide Problemfelder erweisen also, dass es mit Empirie allein nicht getan ist: Es bedarf immer nicht-empirischer bzw. auch pragmatischer Kriterien. Damit blitzt jedoch noch etwas durch, das der Induktivismus ausschliessen wollte und das der deduktiv-hypothetische Ansatz als Problem aufzeigt: ein nicht-normierbares Moment, dem die Kampfansage der Wissenschaftstheorie an die Metaphysik ursprünglich gegolten hatte und das nun zur Selbst-Differenz einer bestimmten Form von Wissenschaftstheorie gehört, die sich eingestehen muss, weder alles rational begründen noch alles auf Empirie aufbauen zu können. Was sich damit zeigt, ist auch für ein allgemeines Verständnis von Wissenschaft relevant: Wissenschaft gleich welcher Art ist sowohl in ihrem internen als auch in ihrem externen Verhältnis selbst-different, das heisst ein komplexes Verbindungs- und Trennungsverhältnis, an dem immer Nicht-Wissenschaftliches, Nicht-Normierbares und letztlich Subjektives mit in Betracht gezogen werden muss. Dies im Auge zu behalten, ist wichtig, wenn es darum geht, einen psychotherapiewissenschaftlich relevanten Wissenschaftsbegriff formulieren zu wollen.

\section{Die Nicht-Eliminierbarkeit des «subjektiven Faktors»}

Eine Gefahrenquelle bei der Begründung des wissenschaftlichen Status von Psychotherapie bzw. auch von Psychotherapiewissenschaft liegt in einem «eingeschränkten Wissenschaftsbegriff» (Leuzinger-Bohleber et al., 2015, S. 112), der unreflektiert Paradigmen der Naturwissenschaft übernimmt und dabei vor allem das Problem nicht lösen kann, wie allgemeine Aussagen über individuell sich ereignende psychische Prozesse gemacht werden können. Plakativ gesagt stehen hier Flexibilität und Determinismus einander entgegen. Dies drückt sich bezüglich der Sprache im Widerspruch zwischen der Mehrdeutigkeit von Begriffen, die gerade als solche eine grösstmögliche Freiheit in der Bedeutungszuschreibung gewährt, und der Forderung nach einer eindeutigen Verwendung von Begriffen im Wissenschaftskontext aus. Das klinisch-theoretische Wissen gilt zudem als subjektbezogen und situationsund kontextabhängig und deshalb als ungeeignet für die Aufstellung allgemein gültiger Aussagen. Besonders seitens psychodynamisch orientierter PsychotherapieRichtungen wird umgekehrt festgestellt, dass quantitative Forschungsdesigns die spezifisch menschliche Dimension der Therapie nicht adäquat abbilden können, dass die Orientierung an der Veränderung von Beschwerden und Symptomen zu oberflächlich sei und dass die eigentliche Veränderung ohnehin im Bereich unbewusster Strukturen liege und deshalb nicht quantifizierbar sei. Genau diese Argumentation wird jedoch von der Gegenseite als Plädoyer für Unwissenschaftlichkeit ins Rennen geführt. 
Wir betreten hier das extrem verminte Feld des LeibSeele-Problems. Von der Seele ist in der modernen Naturwissenschaft nicht viel übrig geblieben. Seele, schreibt Gerhard Roth (2005, S. 37), ist ein "physikalischer Zustand», dessen Gesetze noch nicht hinreichend bekannt sind. Sinn macht die Rede von einer Seele nur mehr - wenn überhaupt - als «Einheit kognitiver, emotionaler und affektiver Zustände und Leistungen». Diese Einheit ist an Gehirnstrukturen und -prozesse gebunden und unterliegt den Naturgesetzen. Interessant ist nun, dass es keine reine Naturwissenschaft geben kann, der es gelingen könnte, das subjektive Moment völlig zu eliminieren bzw. deterministischen Kausalerklärungen zu unterwerfen. Auch die Naturwissenschaft kommt nicht um diesen subjektiven Faktor herum. Dazu zwei kurze Beispiele:

1) Das leitende Paradigma der Neurowissenschaft besagt etwa, dass das Gehirn sich gleichsam in einem dunklen, abgeschlossenen Behälter befindet. Die Sinneseindrücke werden als chemische Impulse durch Neuronen verarbeitet und dadurch wird ein Plan von der Welt und von uns selbst erstellt. Das Ich ist deshalb eigentlich ein Nebenprodukt der Kommunikation des Gehirns mit sich selbst. Ab etwa 100 Milliarden Neuronen soll so etwas wie (Selbst)-Bewusstsein entstehen. Unbeantwortet bleibt: Wie erkennt das Gehirn, dass es überhaupt erkennt, dass es eine Aussenwelt gibt, dass es Gehirn ist? Und wie, dass es so ist, wie die Theorie es darstellt? So entstehen eine Reihe von Paradoxien: Es gibt ein Gehirn in einer Black Box, das die Illusion eines Ichs herausbildet, wobei dieses Ich wiederum eine Vorstellung davon produziert, dass es eigentlich ein Gehirn ist, das sich Vorstellungen von sich macht. Wir haben also feuernde Neuronen, die irgendwann eine Repräsentation ihrer Tätigkeit erstellen, die sich dann fragen kann, wo sie selbst beginnt.

2) Diese paradoxe Verdopplungslogik findet sich auch in der Annahme, dass eine ominöse «Natur» danach streben soll, das Leben über den Tod der Individuen hinaus fortzusetzen. In der populärwissenschaftlich verstandenen Evolutionstheorie heisst es demgemäss, dass es dem «Leben» darum geht, sich selbst zu reproduzieren. Es geht also nicht um Sie oder mich, sondern um eine Art Metasubjekt im Hintergrund, das jedoch paradoxerweise genau uns braucht, um sich selbst erhalten und weiter fortsetzen zu können.

In beiden Beispielen kommt also das ausgeschlossene Subjektive wieder durch die Hintertür ins Spiel, nämlich projiziert auf die «Natur», das Gehirn oder das «Leben». Nun, weder eine verabsolutierte Natur ohne Subjekt noch ein verabsolutiertes Subjekt ohne Natur können befriedigende Lösungen darstellen. Weder kommt das Ich ohne Verbindung zur «Natur» aus noch diese Natur ohne eine Art «subjektiven Faktor». In beiden Fällen - also sowohl dann, wenn sich das Ich über seine Verbindung zur Natur aufzuklären beabsichtigt, als auch dann, wenn sich diese Natur bewusst werden soll - ist der «Stoff», um den es geht, phantasmatisch bzw. selbst-different. Daraus folgt auch, dass der Mensch weder in einer Natur noch im
Geist aufgehen kann. Beschreibungen, die den Menschen auf das eine oder andere beschränken, verfehlen, dass sich sowohl unsere Begriffe von Natur als auch unsere Begriffe von Geist auf phantasmatische Vorannahmen zurückführen lassen, die letztlich nur geglaubt, aber nie bewiesen werden können. Dass jedoch so unterschiedliche Beschreibungen des Menschen überhaupt möglich sind, dass sich also der Mensch von natur- oder geisteswissenschaftlicher Seite her beschreiben kann, könnte freilich als Indiz für Freiheit gelesen werden: für die Freiheit, sich aus unterschiedlichen Perspektiven in ein Selbstverhältnis zu sich selbst und zu anderem setzen zu können. Diese Freiheit könnte auch mit der Erkenntnis einhergehen, dass eine «maximale Selbsttransparenz» nicht zu haben ist, da wir die Umstände des Erkennens nie vollständig erfassen können. Diese Erkenntnis stellt sich gerade auch dann ein, wenn sich eine Wissenschaft eingestehen muss, «noch nicht» alles erklären zu können, wie das eben angeführte Beispiel von Roth zugestehen muss.

\section{Die Selbst-Differenz des subjektiven Faktors}

Vorsicht bezüglich des subjektiven Faktors ist auch geboten, wenn zu seiner Erklärung auf eine besondere Philosophie zurückgegriffen wird, wodurch die Selbst-Differenz dieses subjektiven Faktors verschleiert und in Vorstellungen von Einheit aufgelöst wird. Damit komme ich zu Erismann und zu seiner Bezugnahme auf Fischers Logik der Psychotherapie (2008) und dessen Anleihen bei Hegel zurück. Den dort zu findenden Transfer von Begriffen wie Subjektivität, Objektivität und Intersubjektivität halte ich für nicht geeignet, um eine zeitgemässe Philosophie der Psychotherapiewissenschaft zu unterstützen. Diese Hegel'schen Begriffe sollten nicht einfach dem psychotherapeutischen Prozess übergestülpt bzw. auch nicht als Kriterien der Untermauerung für Wissenschaftlichkeit in der Psychotherapiewissenschaft herangezogen werden, wenn die in ihnen mitschwingenden Selbst-Differenzen nicht entsprechend reflektiert werden. So wird zum Beispiel das Hegel'sche Subjekt unter anderem im Sinne einer Selbstentwicklung eines anonymen Subjekts oder einer anonymen Vernunft, die uns fremd bleibt, verstanden: «als der große Andere eines autistischen Selbst, der übrigens nichts weiter wäre als das phantasmatische Korrelat des Subjekts eines Individualismus des Besitzes und der Sicherheit - zwei Subjekte in des anderen Spiegel, das eine ebenso dumm und böse wie das andere» (Nancy, 2011, S. 92).

Auch wenn sich bei Hegel immer wieder Triaden finden, die nach dem Muster von Objektivität, Subjektivität und Intersubjektivität gedeutet werden können, so ordnet Hegel an anderen Stellen diese Subjektivität wieder der Intersubjektivität über, wodurch die SubjektObjekt-Identität auf eine «intersubjektivitätstheoretische Weiterentwicklung drängt» (Hösle, 1988, Bd. 2, S. 665). Obwohl Hegels System unterschiedliche Deutungsstränge motiviert hat, die für sich gesehen bereits differenzielle Auffassungen von Subjekt, Intersubjektivität etc. repräsentieren, ist es doch 
«in seiner konkreten Form ein endgültiges Vergangenes [...] - den Anspruch, Erkenntnis der Gegenwart zu sein, kann ein System nicht mehr erheben, dem die wichtigsten wissenschaftlichen Entdeckungen, ästhetischen Konzeptionen und historischen Errungenschaften und Gefährdungen der letzten 150 Jahre unbekannt sind» (ebd., Fn. 2).

Bedenken wir dabei auch: Hegel setzt sein System mit der Universalgeschichte und dem Selbstvollzug des Weltgeistes gleich, seine "Apotheose des Staates» bietet eine Legitimierung des Protestantismus und der preussischen Bürokratie, es finden sich rassistische, eurozentristische und speziesistische Züge (Koschorke, S. 76ff.). So sehr die Dialektik ein Prinzip der Unruhe darstellt, «kondensiert» sie doch alle Konflikte und Gegensätze in einem Einheitsbestreben und bietet sich bloss

«als Beschreibungsmittel für Gesellschaften an, die an der Schwelle zur Moderne gerade erst den nötigen Grad an flächendeckender Integration erreicht haben, um sich überhaupt in der Form eines durchgängigen Antagonismus im selben epistemischen Raum mit sich auseinandersetzen zu können» (ebd., S. 80).

Ob dies auch für die uns interessierende Gegenwart und ihre Komplexität möglich ist, ist zu bezweifeln. (Eine komplett entgegengesetzte Meinung vertritt etwa Brandom, 2015).

\section{Zur Selbst-Differenz der Psychotherapiewissenschaft}

Mit diesen kritischen Zeilen möchte ich zu einem Resümee und zu abschliessenden Überlegungen überleiten. Ich habe zu zeigen versucht, dass ein adäquater Wissenschaftsbegriff der Psychotherapiewissenschaft im eigentlichen Sinne weder aus einer Nabelschau der Psychotherapieschulen noch aus einer zu oberflächlichen Auseinandersetzung mit einem gleichzeitig allgemeinen wie eingeschränkten (selbst-differenten) Wissenschaftsbegriff oder aus einem unkritischen Transfer von aus einer eingeschränkten Perspektive stammenden Begrifflichkeit zu gewinnen ist. Versuche dieser Art laufen auf einen Begriff von Psychotherapiewissenschaft hinaus, der reduktiv verstanden und oft inflationär gebraucht wird. Die Lösung sehe ich stattdessen darin, den Theorie- und Begriffsraum so weit zu öffnen, dass die Möglichkeiten wie Unmöglichkeiten unseres Menschseins in all ihren Selbst-Differenzen und wissenschaftlichen Erklärungs- bzw. auch Bemächtigungsversuchen zu Wort kommen können. So würde ein allgemeiner "neutraler Boden» entstehen, der zugleich den normativen Anspruch stellt, reduktive Lösungen nicht zu verabsolutieren und die je eigenen Grundlagen genauer zu reflektieren. Diesen Raum sollte Psychotherapiewissenschaft für sich beziehen und auch gegenüber anderen Diskursen wie etwa der Psychologie, Psychiatrie usw. behaupten.
Mit Hinblick auf dieses umfassendere Panorama unterscheidet sich Psychotherapiewissenschaft als Wissenschaft auch von Psychotherapie. Zwar mögen durchaus wissenschaftliche Tätigkeiten im Zusammenhang mit Psychotherapie geleistet werden (Wirksamkeitsforschung, Metaanalysen etc.). Ich plädiere jedoch dafür, Folgendes zu bedenken (vgl. Burda, 2012): Psychotherapie ist eine bestimmten Methoden folgende Praxis zur Linderung seelischen Leidens, die auf schulen-spezifischen Grundannahmen basiert (Theorie 1. Ordnung). Diese Grundannahmen entstammen unterschiedlichen Wissensfeldern (Medizin, Psychologie, Sozialwissenschaften, Philosophie usw.) und Praktiken (Künste etc.). Verlangt ein avancierter Wissenschaftsbegriff nach einer Reflexion der eigenen Grundlagen (Theorie 2. Ordnung), dann ist Psychotherapie keine Wissenschaft im engeren Sinn. Schulen-intern mag mitunter zwar eine Art Selbstreflexion erfolgen, für die unterschiedliche wissenschaftliche Zugänge gewählt werden. Diese interne Selbstreflexion ist jedoch nicht mit Psychotherapiewissenschaft als externe Perspektive auf Psychotherapie als Theorie und Praxis inklusive ihrer lebensweltlichen, sozio-kulturellen etc. Einbettungen zu verwechseln.

Das Verhältnis Psychotherapie und Psychotherapiewissenschaft stellt in diesem Sinne ein komplexes Verbindungs- und Trennungsverhältnis dar: Psychotherapie und Psychotherapiewissenschaft sind sowohl aufeinander bezogen als auch voneinander zu unterscheiden. Beide entstammen demselben historisch-kulturellen Erfahrungs- und Denk-Raum, bei beiden geht es natürlich um Reflexion und Selbstreflexion. Nur: Der externe Blick der Psychotherapiewissenschaft auf Psychotherapie speist sich aus einer internen Reflexion dieses gemeinsamen Bodens - und bezieht sich damit auf ein viel weiteres Panorama, das von einer als Wissenschaft 1. Ordnung praktizierten Psychotherapie bzw. von einer aus ihr abstrahierten Psychotherapie-Wissenschaft nur teilweise und nicht als solches in den Blick genommen werden kann. Das mögen vielleicht störende bzw. entbehrliche wissenschaftsphilosophische Überlegungen sein: Ihre Relevanz zeigt sich jedoch ganz banal in Form der Tatsache, dass PsychotherapeutInnen zwar wissenschaftlich publizieren können - allein deswegen werden sie jedoch noch keine PsychotherapiewissenschaftlerInnen sein. Ebenso gibt es auch PsychotherapiewissenschaftlerInnen, die keine PsychotherapeutInnen sind und dies auch nicht sein müssen.

Die Akademisierung von Psychotherapie entspringt einem gewissen Zeitgeist, der einerseits eine grundsätzlich zu begrüssende Weiterentwicklung fördert, andererseits jedoch zu gesellschaftspolitischen Anpassungsleistungen zwingt und im Zuge dessen auch zu Vermischungen unterschiedlicher Diskurs- und Praxisfelder führt, die zwar zueinander in einem Verhältnis stehen, jedoch nicht aufeinander reduziert werden sollten. Der um diese Themen bemühte Mainstream könnte meines Erachtens noch einen weiteren Schritt zurück machen, um dadurch die nötige Distanz für die Reflexion der komplexen Verhältnisse gewinnen zu können. Diese Reflexion müsste auch die multiplen Interessen und Motivationen miteinbezie- 
hen, und darüber hinaus noch etwas, das bei unserem Thema latent im Hintergrund mitschwingt und kaum zur Sprache kommt: Ängste, die vor allem die Zukunft betreffen.

Was sich gegenwärtig abzeichnet, sind also unterschiedliche Auffassungen in Bezug auf Psychotherapiewissenschaft. Psychotherapie wird bereits als solche von einigen AutorInnen als «multiparadigmatische» Wissenschaft aufgefasst. Wir hätten so gesehen eine Vielzahl besonderer Psychotherapie-Wissenschaften, die vom eigenen Ansatz her oder aber durch Methoden anderer Wissenschaften reflektiert werden können. Universitäre Einrichtungen bieten deshalb unter dem Etikett Psychotherapiewissenschaft eine parallel laufende psychotherapeutische und wissenschaftliche Ausbildung an. Dies können auch bereits praktizierende oder in Ausbildung begriffene PsychotherapeutInnen in Anspruch nehmen, um «ihre für die Ausbildung ihrer Tätigkeit notwendigen Handlungskompetenzen um vielfältige wissenschaftliche Komponenten und Kompetenzen» ${ }^{4}$ zu erweitern. Psychotherapiewissenschaft wäre so verstanden ein Hybrid - ein bunter Mix unterschiedlicher therapeutischer und wissenschaftlicher Theorien und Methoden. Die Multi-Paradigmatik und Diversität der Psychotherapie-Schulen wäre von einer MultiParadigmatik und Diversität wissenschaftlicher Ansätze durchdrungen und flankiert, die Selbst-Differenz der Psychotherapie würde sich in der Selbst-Differenz der Wissenschaften spiegeln.

Vom Funktionsaspekt her betrachtet ist dies bereits die geübte Praxis. Im Idealfall kann es so auch zu interdisziplinärem Austausch zwischen den Diskursen kommen. Es lassen sich etwa interdisziplinäre PsychoText-Puzzles zusammenstellen und im experimentalbermeneutischen Modell reflektieren, es lassen sich Therapieschulendialoge anregen, für die man einen externen wissenschaftstheoretischen Ansatz bereitstellt. Es lässt sich auch durch eine Nabelschau auf die Psychotherapie das Multi- bzw. Prä-Paradigmatische der Psychotherapie zum Paradigma der Psychotherapiewissenschaft erklären. Was den Begründungs- bzw. Rechtfertigungsaspekt anbelangt lassen sich von einer wissenschaftsphilosophischen Richtung her kommend jedoch sowohl an die eine als auch an die andere Variante Fragen richten, die die multiplen Verbindungen, Differenzen, Primate und Selbstimmunisierungen betreffen - und es lässt sich ein weiterer Horizont anbieten, innerhalb dessen auch die eben skizzierte interne Selbst-Differenz der Psychotherapiewissenschaft angesprochen werden kann.

Ich habe zuvor von einem defizitären und inflationären Gebrauch der Bezeichnung Psychotherapiewissenschaft gesprochen. Das ist natürlich eine Wertung, die allerdings im Bewusstsein dieser Selbst-Differenz operiert, aus der sie sich selbst nicht ausnehmen kann. Deshalb ist es wichtig, nicht nur auf Defizite hinzuweisen, sondern auch auf die mit der Selbst-Differenz gegebenen unterschiedli-

4 So der Werbetext auf www.suttneruni.at (10.06.2019). chen strategischen Ressourcen zur Bewältigung externer Anforderungen, die in Zukunft auf Psychotherapie und Psychotherapiewissenschaft gleichermassen zukommen können.

\section{Literatur}

Barwinski, R. (2017). Intersubjektivität und Selbstreflexion. Psychotherapie-Wissenschaft, 7(1), 23-30.

Brandom, R. B. (2015). Wiedererinnerter Idealismus. Frankfurt/M: Suhrkamp.

Buchmann, R., Schlegel, M. \& Vetter, J. (1996). Die Eigenständigkeit der Psychotherapie in Wissenschaft und Praxis. In A. Pritz (Hrsg.), Psychotherapie - eine neue Wissenschaft vom Menschen (S. 75121). Wien: Springer.

Burda, G. (2012). Formate der Seele. Erkenntnistheoretische Grundlagen und ethische Implikationen der Allgemeinen Psychotherapiewissenschaft. Münster: Waxmann.

Burda, G. (2019). Pandora und die Metaphysica medialis. Psychotherapie - Wissenschaft - Philosophie. Münster: Waxmann.

Carrier, M. (2009). Wissenschaft im Wandel: Ziele, Maßstäbe, Nützlichkeit. Information Philosophie, 3, 16-25.

Erismann, M. (2016). Wissenschaftstheoretische Überlegungen zur Psychotherapiewissenschaft. Psychotherapie-Wissenschaft, 6(1), 6-16.

Erismann, M. (2019; i.d. Heft). Der Wissenschaftsbegriff der Psychotherapiewissenschaft. Psychotherapie-Wissenschaft, 9(2), 13-17.

Fischer, G. (2008). Logik der Psychotherapie. Philosophische Grundlagen der Psychotherapiewissenschaft. Kröning: Ansanger.

Greiner, K. (2012). Psycho-Text-Puzzle und andere Beiträge zu Psychotherapiewissenschaft und Philosophie. Wien: SFU-Verlag.

Greiner, K. (2013). Psycho-Text-Puzzle: Theoriendiskursives Spielverfahren für die Psychotherapiewissenschaft. In F. Lan, F. Wallner \& A. Schulz (Hrsg.), Concepts of a Culturally Guided Philosophy of Science. Contributions from Philosophy, Medicine and Science of Psychotherapy (S. 85-107). Frankfurt/M: Peter Lang.

Habermas, J. (1975). Erkenntnis und Interesse. Frankfurt/M: Suhrkamp. Hösle, V. (1988). Hegels System. Der Idealismus der Subjektivität und das Problem der Intersubjektivität, 2 Bde. Hamburg: Meiner.

Koschorke, A. (2015). Hegel und wir. Frankfurter Adorno-Vorlesungen 2013. Frankfurt/M: Suhrkamp.

Kötter, R. (2017). Wissenschaftstheorie im 20. Jahrhundert. Ein Streifzug durch ihre Geschichte. Aufklärung und Kritik. Vierteljahreszeitschrift der Gesellschaft für Kritische Philosophie, 4, 47-71.

Kuhn, T. (1978). Objektivität, Werturteil und Theoriewahl. In L. Krüger (Hrsg.), Die Entstehung des Neuen (S. 421-445). Frankfurt/M: Springer.

Kuhn, T. (2017). Die Struktur wissenschaftlicher Revolutionen. Frankfurt/M: Suhrkamp.

v. Kutschera, F. (1972). Wissenschaftstheorie. Grundzüge der allgemeinen Methodologie der empirischen Wissenschaften, Bde. I \& II. München: Wilhelm Fink.

Leuzinger-Bohleber, M., Benecke, C. \& Hau, S. (2015). Psychoanalytische Forschung. Methoden und Kontroversen in Zeiten wissenschaftlicher Pluralität. Stuttgart: Kohlhammer.

Nancy, J.-L. (2011). Hegel. Die speculative Anmerkung. Die Unrube des Negativen. Zürich: Diaphanes.

Popper, K. (1979). Ausgangspunkte: meine intellektuelle Entwicklung. Hamburg: Rowohlt.

Roth, G. (2005). Hat die Seele in der Hirnforschung noch einen Platz? In M.F. Peschl (Hrsg.), Die Rolle der Seele in der Kognitions- und Neurowissenschaft. Auf der Suche nach dem Substrat der Seele (S. 27-39). Würzburg: Königshausen \& Neumann.

Sneed, J.D. (1971). The Logical Structure of Mathematical Physics. Dordrecht, Boston, London: D. Reidel Pub. Comp.

Wagner, E. (1996). Psychotherapie als Wissenschaft in Abgrenzung von der Medizin. In A. Pritz (Hrsg.), Psychotherapie - eine neue Wissenschaft vom Menschen (S. 219-247). Wien, New York: Springer. 


\section{Psychotherapy and science: just navel-gazing?}

Referring to the texts «Wissenschaftstheoretische Überlegungen zur Psychotherapiewissenschaft» (2016) and «Der Wissenschaftsbegriff der Psychotherapiewissenschaft» (2019; ibid) by Markus Erismann, the essay investigates the two questions as to what extent psychotherapy sciences can be identified as independent science and whether the «subjective factor» can agree with a general scientific notion. Concerning the first question, we plead for an external scientific approach as opposed to a definition of psychotherapy derived from an excessively self-centered view of psychotherapies. Concerning the second question, we show that science is in general self-differentiating and that even the frowned-upon «subjective factor» cannot be eliminated from the sciences.

Key words: psychotherapy science, scientific theory, science, subject, self-differentiation

\section{Psicoterapia e scienza: contemplazione narcisistica?}

Riassunto: Con riferimento ai testi "Wissenschaftstheoretische Überlegungen zur Psychotherapiewissenschaft» (2016) e «Der Wissenschaftsbegriff der Psychotherapiewissenschaft» (2019; i.d. Heft) di Markus Erismann, il saggio esamina le due domande su come la psicoterapia possa essere identificata come scienza indipendente e se il «fattore soggettivo» sia compatibile con un concetto generale di scienza. Per quanto riguarda la prima domanda, viene sostenuto un approccio scientifico-teorico esterno rispetto a un concetto di psicoterapia scientifica acquisito da una contemplazione narcisistica delle psicoterapie. Per quanto riguarda la seconda domanda, è dimostrato che la scienza è in linea di principio auto-differente (selbst-different) e che persino il malvisto "fattore soggettivo» non può essere eliminato dalle scienze.

Parole chiave: psicoterapia scientifica, teoria della scienza, scienza, soggetto, auto-differenza

\section{Der Autor}

Gerhard Burda ist Philosoph, Psychotherapiewissenschaftler und Lehranalytiker in Wien. Er ist langjähriger Präsident der Österreichischen Gesellschaft für Analytische Psychologie und Mitglied der Wiener Lacan-Schule sowie Autor zahlreicher Veröffentlichungen im Schnittraum von Philosophie, Psychound Medienanalyse; zuletzt erschienen: Psychoanalyse der Erlösung (2016), Mediale Identität/en (2018), Pandora und die Metaphysica medialis (2019).

\section{Kontakt}

Mag. Dr. Dr. habil. Gerhard Burda

Penzinger Str. 69/11

1140 Wien, Austria

E-Mail: comger@gmx.at

www.gerhardburda.com 